Original paper

\title{
Irregular breathing during 4DCT scanning of lung cancer patients: Is the midventilation approach robust?
}

\author{
Marianne C. Aznar ${ }^{\mathrm{a}, \mathrm{b}, *}$, Gitte F. Persson ${ }^{\mathrm{a}}$, Inger M. Kofoed ${ }^{\mathrm{a}}$, Ditte E. Nygaard ${ }^{\mathrm{a}}$, \\ Stine S. Korreman ${ }^{\mathrm{a}, \mathrm{b}, \mathrm{c}}$ \\ a Department of Radiation Oncology, Copenhagen University Hospital, Rigshospitalet, Copenhagen, Denmark \\ ${ }^{\mathrm{b}}$ Niels Bohr Institute, Faculty of Sciences, University of Copenhagen, Denmark \\ ${ }^{\mathrm{c}}$ Department of Science, Systems and Models, Roskilde University, Denmark
}

\section{A R T I C L E I N F O}

\section{Article history:}

Received 10 January 2013

Received in revised form

11 March 2013

Accepted 13 March 2013

Available online 13 April 2013

\section{Keywords:}

4DCT

Breathing patterns

Midventilation

\begin{abstract}
A B S T R A C T
Background: With 4DCT the risk of introducing positional systematic errors in lung cancer radiotherapy can be minimised. A common approach is to plan on the phase bin of the 4DCT best representing the tumour's time-weighted mean position also called the midventilation scan. However breathing irregularities can introduce uncertainties and potentially misrepresent both the tumour trajectory and the determination of the midventilation phase. In this study we evaluated the robustness of the midventilation approach in the presence of irregular breathing patterns.

Methods: A LEGO Mindstorms ${ }^{\circledR}$ phantom with compact balls simulating lung tumours was constructed. The breathing curves loaded in the phantom were either acquired from a human volunteer or constructed with various magnitudes (ranging from 12 to $29 \mathrm{~mm}$ ) as well as various irregularities of motion pattern. Repeated 4DCT scans were performed while tumour trajectories were recorded with two motion tracking systems.

Results: The time-weighted mean tumour position is accurately represented in 4DCT scans, even for irregular breathing patterns: the position presentation in the midventilation scan was always within in one standard deviation of the global position presentation $(3 \mathrm{~mm}$ and $2 \mathrm{~mm}$ for regular and irregular breathing patterns, respectively). The displacement representation tended to be underestimated in 4DCT scans.

Conclusion: The midventilation approach is robust even in the presence of breathing irregularity. The representation of the tumour trajectory in 4DCT scans is affected by breathing irregularity and the extent of tumour motion can be underestimated, which will affect the calculation of patient-individualised margins based on the 4DCT scan.
\end{abstract}

๔ 2013 Associazione Italiana di Fisica Medica. Published by Elsevier Ltd. All rights reserved.

\section{Introduction}

Imaging of moving tumours represents a major challenge in modern radiotherapy. Conventional CT (3DCT) only gives a snapshot of the tumour in an arbitrary phase of the respiratory cycle. Positional uncertainties translate into systematic errors impacting precision throughout the radiotherapy course.

Breathing-correlated computer tomography (4DCT) provides several time-resolved images and thereby a representation of the motion trajectory of the tumour during the full respiratory cycle [1-5].

\footnotetext{
* Corresponding author. Department of Radiation Oncology, Copenhagen University Hospital, Rigshospitalet, 2100 Copenhagen, Denmark. Tel.: +4535454830.

E-mail addresses: marianne.aznar@regionh.dk, marianne.aznar@rh.regionh.dk (M.C. Aznar).
}

In radiotherapy planning, it is crucial that the planning scan mimics the treatment situation as accurately as possible. In the absence of gating or tracking, a moving tumour is best represented in its most likely position, i.e. a time-weighted average position. With 3DCT this can not necessarily be achieved as tumours can be captured at any point of the breathing cycle, including endinspiration or end-expiration. With 4DCT the risk of introducing positional systematic errors can be minimised, for example by planning on the phase bin of the 4DCT best representing the tumours time-weighted mean position: the midventilation scan (MidV) [6]. The tumour's intrafraction motion span as measured in the 4DCT scan can then be incorporated into a probabilistic calculated planning target volume (PTV) margin $[7,8]$ as a random uncertainty. Planning on the MidV can lead to a reduction of the required PTV margin when compared to 3DCT-based planning $[9,10]$. 
However, 4DCT is also subject to uncertainties [11], in particular if the patient breathes irregularly. The impact of breathing irregularity on the image quality in 4DCT scans has been reported in terms of shape and position representation [12-15] and characterisation of artefacts [16] but the impact on the representation of the tumour trajectory and especially on the determination of the midventilation phase has not been fully explored. An incorrect determination of the midventilation phase can potentially introduce systematic errors in the treatment planning process.

In this phantom study we evaluated how well 4DCT represents the tumour trajectory with both regular and irregular breathing patterns. We also investigated the sensitivity of the determination of the midventilation phase to irregular breathing: can the tumour position on the midventilation phase safely be assumed to be representative of the "true" time-weighted average tumour position if a patient breathes in a very irregular pattern? We tested this property, here called "robustness" of the midventilation approach, in the presence of breathing patterns of varying degrees of irregularity and of varying motion spans.

\section{Materials and methods}

\section{Phantom construction}

For this study, we used an in-house developed programmable motion phantom (shown in Fig. 1) built from LEGO Mindstorms ${ }^{\circledR}$ nxt (LEGO, Billund, Denmark). This motion phantom was combined with two commercially available motion detection systems: ExacTrac (BrainLAB) and RPM (Varian Medical Systems), see below. The LEGO phantom allows for flexible construction opportunities and enables rather sophisticated computer control of built-in motors. Most parts are made from plastic and thus will not cause metal imaging artefacts in CT-scans.

The phantom consisted of a part simulating the breathing motion of the chest wall in $z$-direction (equivalent to the AP direction in the scan plane, see Fig. 1), and another part simulating two lung tumours capable of moving both in the $z$-direction and $y$-direction (table feed direction). One motor controlled the motion of the "chest wall" part of the phantom and two other motors controlled the motion of the two "tumours" - one in shape of a hollow plastic ball filled with sesame seeds (diameter $4 \mathrm{~cm}$ ) and the other a

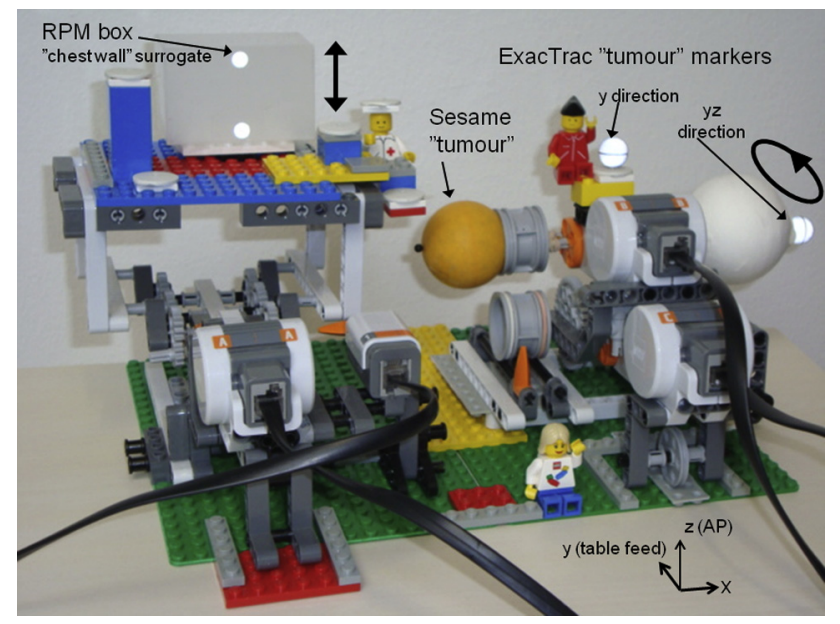

Figure 1. The LEGO phantom including the RPM marker box moving in vertical (anterior-posterior) direction $(z)$, and the balls and attached ExacTrac markers moving in horizontal ( $y$ or table feed) and vertical ( $z$ or AP) directions. compact paper ball (diameter $5 \mathrm{~cm}$ ). The displacement in the $y$-direction could be changed to reflect the depth of each breath by means of a railway construction: one of the motors controlled the longitudinal motion ( $y$-direction), rolling back and forth on the rails, while the other motor could rotate the balls to get a simultaneous motion in the $z$-direction. Only the motion in the $y$-direction (table feed) was adjustable while the motion in $z$-direction (AP) was constant: $12 \mathrm{~mm}$ for every breathing cycle. The speed of the tumour motion was constant throughout the study (about $2.5 \mathrm{~mm} / \mathrm{s}$ ). The motion of the "chest wall" platform in the $z$-direction and the displacement of the balls in the $y$-direction were synchronised.

\section{Motion monitoring}

Two different systems were used to track and record the motion. The Real-time Position Management ${ }^{\mathrm{TM}}$ (RPM) 1.7 system (Varian Medical Systems Inc, Palo Alto, USA) consists of a small plastic box with two reflecting markers and a camera with infrared emitting diodes connected to a computer. The RPM marker box was placed on the "chest wall" platform (Fig. 1) while the camera was attached to the couch of the CT scanner. The RPM system was connected to the CT scanner and beam-on time was registered. This system gives the relative motion data and was only used for reconstruction of the 4DCT, emulating an external breathing signal from a moving chest wall.

The ExacTrac (BrainLAB, Feldkirchen, Germany) uses small reflecting marker spheres and two cameras stereoscopically recording the motion from two different angles. It has a submillimetre precision and can be calibrated to an isocentre to generate absolute coordinates of the markers. Two ExacTrac markers were placed on the tumour part of the phantom: one on the paper ball to give the coordinates of the full motion in both $\mathrm{y}$ - and z-directions and one on the motor to give coordinates of the motion in the $y$-direction only (Fig. 1). The ExacTrac data was used in the position accuracy analysis to track the actual 3D trajectory of the tumours during the scan.

In this study, the positions recorded by ExacTrac were considered to represent the true tumour position and used as a reference for the tumour position observed on the CT images. Hence, no assumptions were made as to the performance of the LEGO phantom itself. To correlate positions recorded with the ExacTrac system to positions read out from the scans (DICOM coordinates), a reference cross made of soldering wire was placed $1 \mathrm{~cm}$ below the isocentre to which the ExacTrac was calibrated. The cross was visible in the scans and enabled correlation of the coordinates of ExacTrac to the coordinates in the scan.

\section{Breathing patterns}

Six breathing curves were constructed for the phantom with the LEGO software: three regular breathing curves simulating displacements of respectively 12,20 and $29 \mathrm{~mm}$ at constant speed of the balls in the $y$-direction; and three irregular breathing curves (var1, var2 and var3) where the displacement of the balls in the $y$-direction changed randomly between 12 and $29 \mathrm{~mm}$ from cycle to cycle (illustrated in sub Fig. 2a). With the constructed breathing motion curves, the phantom moved the balls in both $y$ - and z-directions (motion trajectories are shown in Fig. 4). The duration of the constructed breathing cycles ranged from 4 to $8 \mathrm{~s}$ (except in very irregular patterns, where cycle durations up to $12 \mathrm{~s}$ were present).

Additionally, two human breathing curves from a healthy volunteer (IMK) were recorded with the RPM system: a regular human breathing curve consisting of three repeats of a short 
a)

Phantom breathing curves, varying displacement

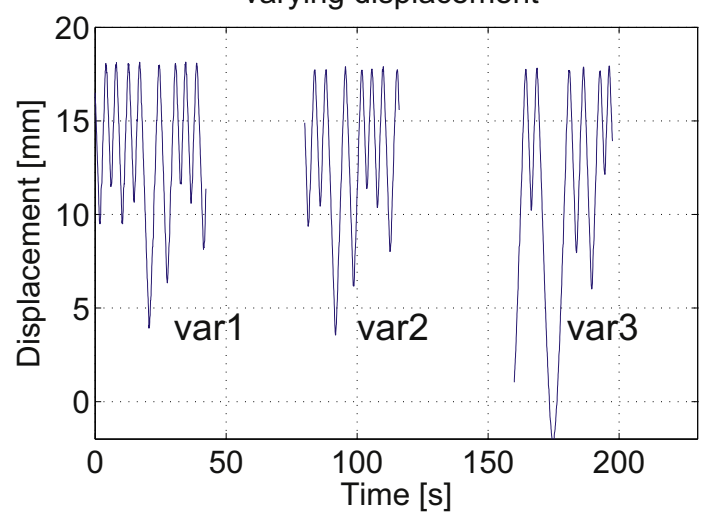

b)
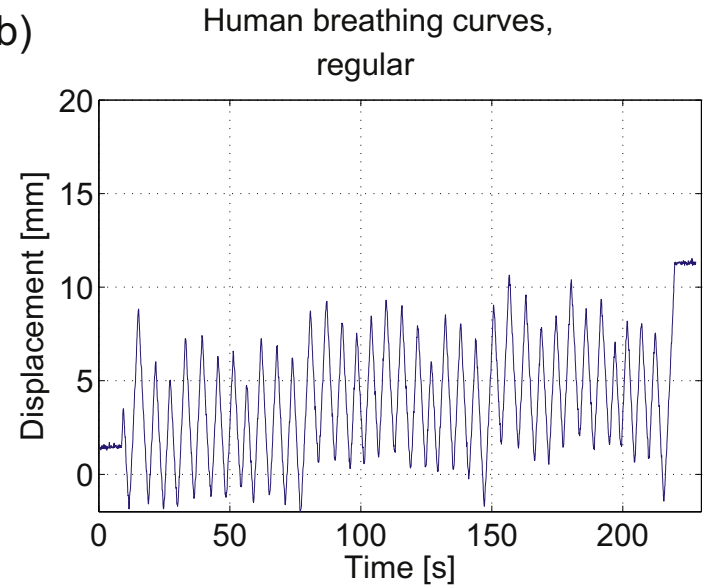

c)

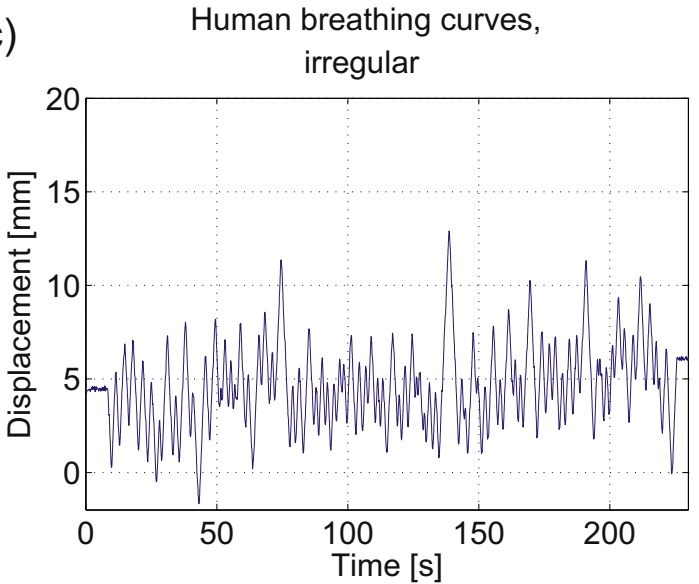

Figure 2. Breathing curves used for the experiments: a) Three curves created using the phantom programming software with varying displacements, b) A human breathing curve with high degree of regularity, c) A human breathing curve with high degree of irregularity.

sequence where the volunteer was breathing quietly (210 s in total) and an irregular human breathing curve consisting of one long recorded sequence where the volunteer purposefully breathed with an irregular pattern (220 s), shown in sub Fig. 2 b and c. For these human breathing curves, the breathing cycle durations could be considerably shorter (down to $2 \mathrm{~s}$ in the irregular breathing pattern) and due to limitations in the speed and rotation settings of the LEGO motors, the balls only moved in the $y$-direction (table feed direction).

\section{CT scans}

All scans were acquired in helical mode using a Siemens Sensation Open (Siemens Medical Solutions, Erlangen, Germany) multislice CT. Clinical protocols were used and 4DCT scans were acquired with a pitch of 0.1 , a rotation time of $1 \mathrm{~s}$ and a slice thickness of $3 \mathrm{~mm}$. Ten bins were used for the reconstruction, each corresponding to a phase percentage of the breathing cycle. The bins were reconstructed at: $0 \%$ (peak inspiration), 10\%, 20\%, 30\%, $40 \%, 50 \%, 60 \%, 70 \%, 80 \%$, and $90 \%$ of each respiratory cycle.

The sesame "tumour" was manually contoured in Eclipse with fixed window level settings in each phase of the 4DCT scans. The coordinates of the ExacTrac markers and of the soldering wire cross were registered on all scans manually using Eclipse measurement tools. The uncertainty in reading out the DICOM coordinates in Eclipse (Varian) and correlating the Eclipse and ExacTrac coordinate systems was estimated to be $\sim 2 \mathrm{~mm}$ in the $x$ and $y$ (in-plane) directions. This includes the error in correlating the ExacTrac and Eclipse coordinate systems, the position uncertainty of the box related to the soldering wire cross as well as the read-out procedure from Eclipse taking into account the limited resolution of the screen. Hence, deviations from the recorded position greater than $2 \mathrm{~mm}$ can most likely be attributed to motion artefacts.

Before further processing, a translation of the DICOM coordinates to ExacTrac coordinates was done. For all 4DCT scans, the midventilation scan was found by calculating the mean of the coordinates of the centre of the volume of the delineated sesame ball in Eclipse to give the time-weighted average position of the ball (since all bins were equally spaced in time). The phase in the 4DCT scan with ball coordinates closest in space to this mean position was then selected as the midventilation scan.

\section{Trajectory analysis in constructed breathing curves}

For analysis of trajectories with constructed breathing curves, the position of the ExacTrac marker (on the paper ball) in the 3Dand 4DCT scans was compared with the position recorded by the ExacTrac system itself during the acquisition of the scans. The recorded positions by the ExacTrac system (with a frequency of $20 \mathrm{~Hz}$ ) were compared to the coordinates read out from the bins of the 4DCT scan by determining the ExacTrac data corresponding to the projection data used for the reconstruction of each 4DCT bin and averaging all positions corresponding to one phase bin. The marker position in the scan bins was compared to the mean position of all the recorded data points for that bin and residuals were calculated for both directions of motion. The midventilation scan is determined as described in Section 2.4 and compared to the timeweighted average marker position recorded by ExacTrac.

\section{Trajectory analysis in human breathing curves}

For analysis of trajectories with human breathing curves, the motion of the ExacTrac marker on the motor was used (only motion in the $y$-direction). The human breathing curves were used to test the ability of the 4DCT scan to represent the full span of a patient's breathing ("global breathing curve") as opposed to the patient's breathing during the short span (about $40 \mathrm{~s}$ ) of the beam-on time of the scanner (called "local breathing curve"). Seven 4DCT scans were performed with each of the two human breathing curves and the CT acquisition times were distributed evenly along the breathing motion curves. In order to assess how well the "local breathing curves" were representative of the "global breathing curves" a frequency plot was generated to compare the recorded position distribution of the marker during the full breathing curve ("global position distribution") and the sub-section of the curve during each 
a)

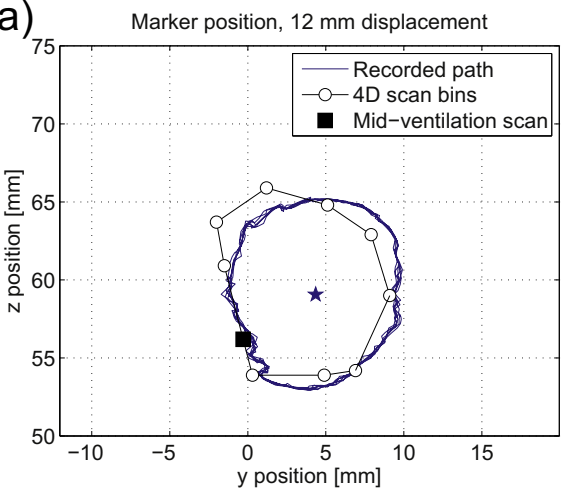

c)

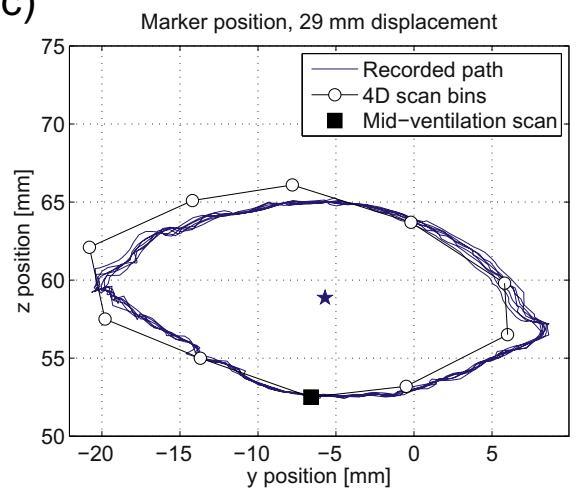

e)

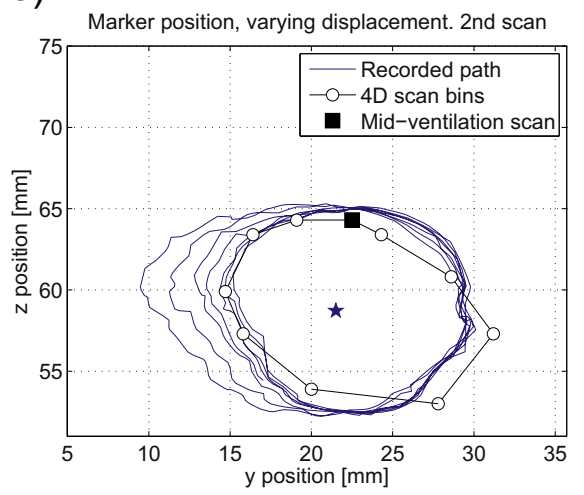

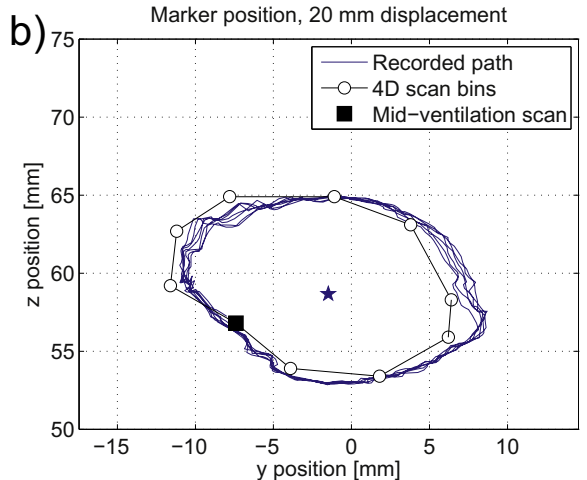

d)

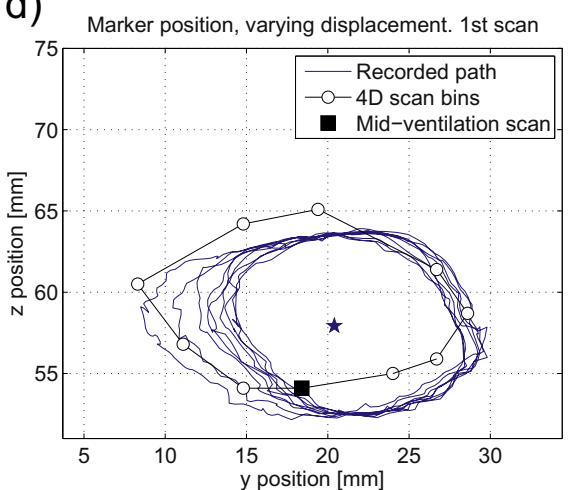

f)

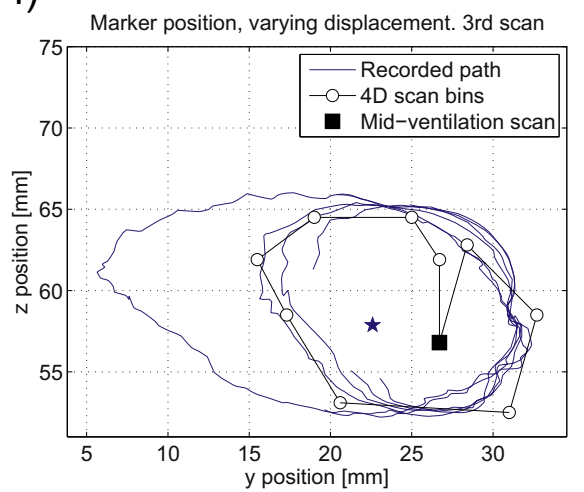

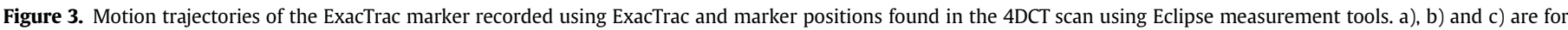

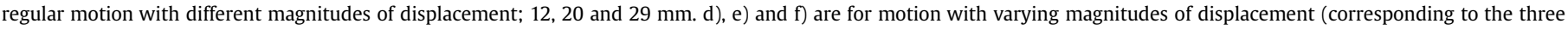
curves in Fig. 2a). The star symbol represents the time-weighted average marker position from the ExacTrac-recorded data.

scanner beam-on time ("local position distribution"). The rendition of the global breathing motion by 4DCT scans was then investigated by comparing the mean positions of the marker as well as the standard deviation of the marker position in the global position distribution and in each of the 4DCT scans.

Since the speed of the tumour marker was constant, a perfectly periodic breathing would in this case be represented as an equal distribution for all positions in the frequency plot. The position of the tumour marker in each midventilation scan was also represented in the frequency plot, and compared to the time-averaged marker position as recorded by ExacTrac.

\section{Results}

\section{Trajectory representation for constructed breathing curves}

Figure 3 shows the path of the ExacTrac marker as recorded by the ExacTrac system versus the path as derived from the marker's coordinates in 4DCT scan for the constructed breathing curves. The regular displacements measured by ExacTrac were about $1 \mathrm{~mm}$ shorter than those provided to the phantom $(11.1 \mathrm{~mm}$, $19.2 \mathrm{~mm}$ and $28.7 \mathrm{~mm}$ respectively compared to 12,10 and $29 \mathrm{~mm}$ ) due to the limited precision of the motors. For comparison, the respective displacements measured from the 4DCT data were $11.1 \mathrm{~mm}, 18 \mathrm{~mm}$ and $26.8 \mathrm{~mm}$. For regular breathing curves, there was a good position representation with 4DCT scans, as the position residuals were not exceeding the uncertainty level of $2 \mathrm{~mm}$ markedly (maximum residual of $1.9,2.5$ and $1.1 \mathrm{~mm}$ in the $x, y$ and $z$ directions, respectively). Moreover, the extent of displacement did not have an impact on the position representation as long as the displacement was regular. However, with the irregular breathing curves (Fig. $3 \mathrm{~d}-\mathrm{f}$ ) the discrepancy between the ExacTrac recorded positions and the position representation in the 4DCT scan bins was more pronounced: the residuals for some bins were considerably larger, up to $6 \mathrm{~mm}$ in both $y$ and $z$ directions. 
a) Human breathing curve distributions, regular

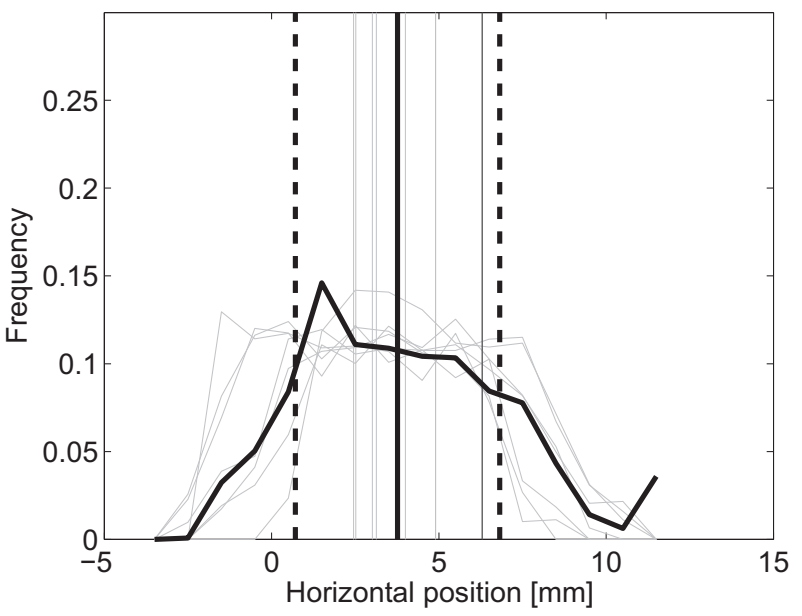

b) Human breathing curve distributions, irregular

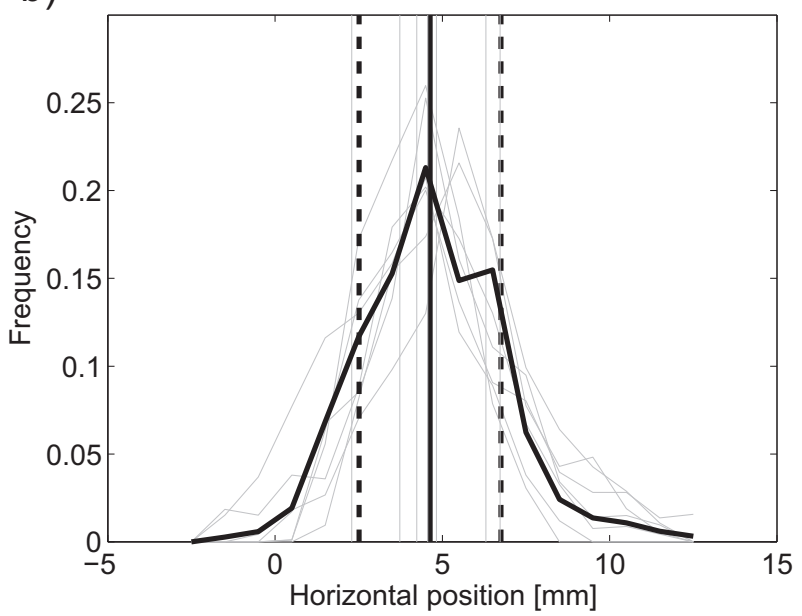

Figure 4. Position distribution histograms for the human breathing curves. The global distributions are shown in thick lines, and the distributions for only the time intervals of repeated 4DCT scan recordings are shown in thin lines. The vertical thick lines indicate the global mean position (solid) and one standard deviation (dashed). The vertical thin lines indicate the marker position in each midventilation scan.

For the largest irregular displacement (var3, see Fig. 2-left for the breathing curve, and Fig. 3f for the influence on the trajectory representation) the midventilation scan did not represent a true position on the tumour's trajectory as measured with ExacTrac due to the large variation in displacement between each breathing cycle. However the midventilation position representation is still closer to the time-weighted average of the ExacTrac recorded positions (blue star symbol) than the tumour position representation in any other 4DCT scan bin.

\section{Trajectory representation for human breathing curves}

Figure 4 shows the position distribution histograms for the ExacTrac marker placed on the motor (representing displacement in the $y$-direction) with the regular and irregular human breathing curves during consecutive 4DCT scans (represented by thin lines), and for the entire curves (represented by thick lines). The mean position of the global position distribution is indicated as a thick line, while the standard deviation (SD) is displayed as thick dashed lines. The regular breathing curve display a flatter and broader distribution of position, as all breathing cycles have almost the same amplitude (i.e. the breathing pattern is more "regular"). On the other hand the irregular breathing pattern displays a narrow position distribution, as the amplitudes of cycles can vary considerably. Note that the SD of the global positions is greater for regular breathing than for irregular breathing because the regular human breathing pattern was deeper (larger mean displacement) than the irregular breathing pattern in this experiment. In Table 1 the SD of the global positions of the marker and the SD of the positions of the marker throughout the bins of the 4DCT scans are listed. These data indicate that the global position distribution is better represented in scans from regular human breathing than from irregular human breathing, which is not unexpected. For the irregular human breathing the SDs of the marker positions in the 4DCT scans are considerably smaller than the SD of the global positions: this suggests that the scans acquired during irregular breathing underestimate the total displacement of the marker. Hence, for a patient with irregular breathing, this extent of the tumour displacement may be underestimated in 4DCT scanning.

The positions of the marker in each of the midventilation scans for the consecutive 4DCT scans are also represented in Fig. 4 (solid thin lines) and fall within 1 SD of the mean global position for regular human breathing, and close to within 1 SD for irregular human breathing. This suggests that the time-weighted mean position of the tumour is well represented in 4DCT scans, even for irregular breathing. However, the span of the tumour motion might be underestimated.

\section{Discussion}

Breathing regularity is crucial for the accurate representation of the tumour trajectory: the representation of position distribution was increasingly degraded for increasing degrees of breathing irregularity. The consequence is a risk that the extent of tumour motion might be underestimated in 4DCT of patients with irregular breathing and this error will negatively affect the calculation of patient-individualised margins. Richter at al. [14] have reported that a $4 \mathrm{DCT}$ represents at best the mean extent of the tumour displacement, but not the maximum extent of tumour motion in presence of irregular breathing. Furthermore, there is a risk that the position found from a 4DCT scan midventilation approach may not represent the actual mean position. Hence caution is generally advised when using 4DCT scans and devising individualised margins for patients with noticeably irregular breathing. Nevertheless, the results of this study suggest that, even for irregular breathing, the representation of the tumour position in the midventilation scan of a 4DCT is accurate and representative of the patient's global breathing pattern. If one considers that the scan bins of a 4DCT scan might represent the possible distribution of 3DCT scans acquired at random times of the patient breathing cycle, it becomes clear that the midventilation approach can determine the average tumour position as least as accurately, or better than, a 3DCT approach.

Repeated 4DCT scans can lead to different representations of the tumour position in the midventilation bins: Fig. 4 suggests that

Table 1

Standard deviation of the position distribution of the marker in 4DCT scans using human breathing curves. BC: breathing curve.

\begin{tabular}{cllllllll}
\hline & $\begin{array}{l}\text { Global } \\
\text { BC }\end{array}$ & $\begin{array}{l}\text { Scan } \\
1\end{array}$ & $\begin{array}{l}\text { Scan } \\
2\end{array}$ & $\begin{array}{l}\text { Scan } \\
3\end{array}$ & $\begin{array}{l}\text { Scan } \\
4\end{array}$ & $\begin{array}{l}\text { Scan } \\
5\end{array}$ & $\begin{array}{l}\text { Scan } \\
6\end{array}$ & $\begin{array}{l}\text { Scan } \\
7\end{array}$ \\
\hline $\begin{array}{c}\text { SD for the regular } \\
\text { pattern (mm) } \\
\begin{array}{c}\text { SD for the irregular } \\
\text { Pattern (mm) }\end{array}\end{array}$ & 3.06 & 2.90 & 2.83 & 2.95 & 2.74 & 2.90 & 2.50 & 2.97 \\
\hline
\end{tabular}


differences in tumour position between several midventilation scans can be up to $5 \mathrm{~mm}$. However, since all these estimates of the midventilation position fall within one standard deviation of the "true" global mean position and provided that this uncertainty is included in the treatment margin calculation, this does not represent a contra-indication to the midventilation approach. Our data indicate therefore that the midventilation approach is robust even in presence of irregular breathing.

It has been reported that patient breathing and tumour motion can vary significantly both during a single treatment fraction, but also and more importantly between treatment fractions $[17,18]$. The question of whether a 4DCT scan represents the global breathing pattern (throughout the whole course of treatment) is then essential, and should ideally be coupled with $4 \mathrm{D}$ image verification strategies at the treatment machine.

Other groups have commented on the influence of breathing irregularity on tumour presentation and risk of artefacts and have concluded that displacement-based binning is less sensitive to breathing irregularity than phase-based binning [12,19], as amplitude binning has the ability to provide smaller residual motion within the bins. However, amplitude binning may undersample images in the bins where large motion is present within short time leading to interpolation artefacts in the affected bins. Regardless of binning method, the risk of artefacts will remain present and lead to uncertainties in delineation [20-22] which should be accounted for when $4 \mathrm{DCT}$ data are used for treatment planning.

In cases with very irregular breathing, coaching, either visual or audio, may be used to improve breathing regularity $[23,24]$ but will not reduce interfraction variability in amplitude [25]. However, newer combined audiovisual coaching strategies using bio-feedback mechanisms are promising [26]. The usefulness of abdominal compression in order to reduce tumour motion is debated [27].

This study is limited by several factors. First, the LEGO phantom is home-made and its performance (i.e. how close the tumour trajectory corresponds to the breathing curve data provided to the phantom's motors) has not been thoroughly tested. Our data suggest that the LEGO phantom provides shorter displacements than programmed. However, in this context, the phantom's performance will not affect the conclusions of the study, as the tumour position is recorded through the ExacTrac markers and no "expected" trajectory is used in the analysis. Second, due to some limitations of the LEGO motors, the motion is unidirectional while commercial 4D-phantom systems are now available which can represent a 3D tumour motion. Though lung tumours move predominantly in the superior-inferior direction and our simulation represents the most common clinical scenario, it cannot be excluded that in some clinical situations, other directions of motion (or complex motion patterns, such as hysteresis) may impact the midventilation position. Third, we only used one scanner type and one scanning protocol: different acquisition settings might influence the results. Finally, this study only addresses the determination of the midventilation phase and tumour position: it does not address potential image artefacts or deformation of the tumour volume within the midventilation phase. In our institution, a short breath-hold CT is usually acquired after each clinical 4DCT scan, in order to obtain a "static" image of the tumour: this image is then used to check that the scan chosen as the midventilation phase does not include major deformations or artefacts which could affect the delineation of the target volume and the resulting treatment plan.

\section{Conclusion}

The results of this study demonstrate that the midventilation approach is robust even in the presence of irregular breathing: the time-weighted mean position of the tumour from a 4DCT is within one standard deviation of the mean position of the global breathing pattern of the patient, and is at least as accurate as the position that would be determined from a 3DCT scan. However, as expected, the span of tumour motion can be underestimated from a single 4D-CT exam in the presence of irregular breathing and this will affect the calculation of patient-individualised margins.

\section{References}

[1] Keall P. 4-dimensional computed tomography imaging and treatment planning. Semin Radiat Oncol 2004;14:81-90.

[2] Ford EC, Mageras GS, Yorke E, Ling CC. Respiration-correlated spiral CT: a method of measuring respiratory-induced anatomic motion for radiation treatment planning. Med Phys 2003;30:88-97.

[3] Low DA, Nystrom M, Kalinin E, Parikh P, Dempsey JF, Bradley JD, et al. A method for the reconstruction of four-dimensional synchronized CT scans acquired during free breathing. Med Phys 2003;30:1254-63.

[4] Pan T, Lee TY, Rietzel E, Chen GT. 4D-CT imaging of a volume influenced by respiratory motion on multi-slice CT. Med Phys 2004;31:333-40.

[5] Vedam SS, Keall PJ, Kini VR, Mostafavi H, Shukla HP, Mohan R. Acquiring a four-dimensional computed tomography dataset using an external respiratory signal. Phys Med Biol 2003;48:45-62.

[6] Wolthaus JW, Schneider C, Sonke JJ, van HM, Belderbos JS, Rossi MM, et al. Mid-ventilation CT scan construction from four-dimensional respirationcorrelated CT scans for radiotherapy planning of lung cancer patients. Int J Radiat Oncol Biol Phys 2006;65:1560-71.

[7] van Herk M, Remeijer P, Rasch C, Lebesque JV. The probability of correct target dosage: dose-population histograms for deriving treatment margins in radiotherapy. Int J Radiat Oncol Biol Phys 2000;47:1121-35.

[8] McKenzie AL, van HM, Mijnheer B. The width of margins in radiotherapy treatment plans. Phys Med Biol 2000;45:3331-42.

[9] Wolthaus JW, Sonke JJ, van HM, Belderbos JS, Rossi MM, Lebesque JV, et al. Comparison of different strategies to use four-dimensional computed tomography in treatment planning for lung cancer patients. Int J Radiat Oncol Biol Phys 2008;70:1229-38.

[10] Korreman S, Persson G, Nygaard D, Brink C, Juhler-Nottrup T. Respirationcorrelated image guidance is the most important radiotherapy motion management strategy for most lung cancer patients. Int J Radiat Oncol Biol Phys 2012;83:1338-43.

[11] Hurkmans CW, van Lieshout M, Schuring D, van Heumen MJ, Cuijpers JP, Lagerwaard FJ, et al. Quality assurance of 4D-CT scan techniques in multicenter phase III trial of surgery versus stereotactic radiotherapy (radiosurgery or surgery for operable early stage (stage $1 \mathrm{~A}$ ) non-small-cell lung cancer [ROSEL] study). Int J Radiat Oncol Biol Phys 2011;80:918-27.

[12] Sarker J, Chu A, Mui K, Wolfgang JA, Hirsch AE, Chen GT, et al. Variations in tumor size and position due to irregular breathing in 4D-CT: a simulation study. Med Phys 2010;37:1254-60.

[13] Noel CE, Parikh PJ. Effect of mid-scan breathing changes on quality of 4DCT using a commercial phase-based sorting algorithm. Med Phys 2011;38:2430-8.

[14] Richter A, Wilbert J, Flentje M. Dosimetric evaluation of intrafractional tumor motion by means of a robot driven phantom. Med Phys 2011;38:5280-9.

[15] Guckenberger M, Wilbert J, Krieger T, Richter A, Baier K, Flentje M. Midventilation concept for mobile pulmonary tumors: internal tumor trajectory versus selective reconstruction of four-dimensional computed tomography frames based on external breathing motion. Int J Radiat Oncol Biol Phys 2009;74:602-9.

[16] Han D, Bayouth J, Bhatia S, Sonka M, Wu X. Characterization and identification of spatial artifacts during 4D-CT imaging. Med Phys 2011;38:2074-87.

[17] Sonke JJ, Lebesque J, van Herk M. Variability of four-dimensional computed tomography patient models. Int J Radiat Oncol Biol Phys 2008;70:590-8.

[18] Juhler NT, Korreman SS, Pedersen AN, Aarup LR, Nystrom H, Olsen M, et al. Intra- and interfraction breathing variations during curative radiotherapy for lung cancer. Radiother Oncol 2007;84:40-8.

[19] Li H, Noel C, Garcia-Ramirez J, Low D, Bradley J, Robinson C, et al. Clinical evaluations of an amplitude-based binning algorithm for 4DCT reconstruction in radiation therapy. Med Phys 2012;39:922-32.

[20] Persson GF, Nygaard DE, Brink C, Jahn JW, Munck af RP, Specht L, et al. Deviations in delineated GTV caused by artefacts in 4DCT. Radiother Oncol 2010;96:61-6.

[21] Persson GF, Nygaard DE, Munck af Rosenschold P, Vogelius IR, Josipovic M, Specht L, et al. Artifacts in conventional computed tomography (CT) and free breathing four-dimensional CT induce uncertainty in gross tumor volume determination. Int J Radiat Oncol Biol Phys 2011 Aug 1;80(5):1573-80.

[22] Yamamoto T, Langner U, Loo Jr BW, Shen J, Keall PJ. Retrospective analysis of artifacts in four-dimensional CT images of 50 abdominal and thoracic radiotherapy patients. Int J Radiat Oncol Biol Phys 2008;72:1250-8.

[23] George R, Chung TD, Vedam SS, Ramakrishnan V, Mohan R, Weiss E, et al Audio-visual biofeedback for respiratory-gated radiotherapy: impact of audio instruction and audio-visual biofeedback on respiratory-gated radiotherapy. Int J Radiat Oncol Biol Phys 2006;65:924-33.

[24] Kini VR, Vedam SS, Keall PJ, Patil S, Chen C, Mohan R. Patient training in respiratory-gated radiotherapy. Med Dosim 2003;28:7-11. 
[25] Persson GF, Nygaard DE, Olsen M, Juhler-Nottrup T, Pedersen AN, Specht L, et al. Can audio coached 4D CT emulate free breathing during the treatment course? Acta Oncol 2008;47:1397-405.

[26] Venkat RB, Sawant A, Suh Y, George R, Keall PJ. Development and preliminary evaluation of a prototype audiovisual biofeedback device incorporating a patient-specific guiding waveform. Phys Med Biol 2008;53: N197-208

[27] Bouilhol G, Ayadi M, Rit S, Thengumpallil S, Schaerer J, Vandemeulebroucke J, et al. Is abdominal compression useful in lung stereotactic body radiation therapy? A 4DCT and dosimetric lobe-dependent study. Phys Med 2013;29(4):333-40. 